\title{
REU Site: Bio-Grid Initiatives for Interdisciplinary Research and Education ${ }^{\text {th }}$
}

\author{
Chun-Hsi Huang \\ Computer Science 8 Engineering, University of Connecticut, Storrs, CT 06269, USA \\ chunhsi.huang@uconn.edu
}

\begin{abstract}
${ }^{2}$ Extended from an earlier version that appeared in Proceedings of the Workshop on Education for High-Performance Computing (EduHPC), Nov. 15-20, 2015, Austin, TX.
\end{abstract}

Preprint submitted to Journal of Parallel and Distributed Computing

January 5, 2017

(C) 2017. This manuscript version is made available under the Elsevier user license http://www.elsevier.com/open-access/userlicense/1.0/ 


\title{
REU Site: Bio-Grid Initiatives for Interdisciplinary Research and Education ${ }^{\text {tr }}$
}

\author{
Chun-Hsi Huang \\ Computer Science \& Engineering, University of Connecticut, Storrs, CT 06269, USA \\ chunhsi.huang@uconn.edu
}

\begin{abstract}
Recently more and more universities have been incorporating HPC (High Performance Computing) in their computing curriculum [1]. The Bio-Grid REU (Research Experience for Undergraduates) Site offers undergraduate students interested or experienced in HPC a summer research opportunity to participate in projects that apply HPC in various life-science disciplines. The projects are associated with the Bio-Grid Initiatives conducted at the University of Connecticut. Training seminars are designed to equip students with background knowledge such as basic parallel programming, large-scale data analytics, and middleware support, etc., as well as some ongoing projects using these computing methods. Students participate in several collaborative projects supported by a campus-wide computational and data grid. The REU project introduces such interdisciplinary research work to students in the early stage of their academic career to spark their interest. The project aims at preparing future software engineers to formalize and solve emerging life-science problems, as well as lifescience researchers with a strong background in high-performance computing. The Bio-Grid REU Site was supported by the National Science Foundation from 08-10 and 12-14, with a website located at http://biogrid.engr.uconn.edu/REU Keywords: HPC, Computational Biology, Grid and Cloud Computing
\end{abstract}

\footnotetext{
${ }^{27}$ Extended from an earlier version that appeared in Proceedings of the Workshop on Education for High-Performance Computing (EduHPC), Nov. 15-20, 2015, Austin, TX.
}

Preprint submitted to Journal of Parallel and Distributed Computing

January 5, 2017 


\section{Instruction}

Research work in life sciences increasingly relies on globally distributed information and knowledge repositories. The quality and performance of future computing and storage infrastructure in support of such research depends heav5 ily on the ability to exploit these repositories, to integrate these resources with local information processing environments in a flexible and intuitive way, and to support information extraction and analysis in a timely and on-demand manner.

With an ever-increasing throughput, the next-generation sequencing technologies have led to generate a large amount of DNA data, to the extent that in its first six months, next-generation sequencing outpaced what already have been generated in the past 21 years by old technologies 2. This wealth of genomic information dramatically accelerates progress toward a comprehensive understanding of the genetic mechanisms involved in diverse biochemical processes pertinent to bioremediation, medicine, biotechnology and agriculture. Efficiency and accuracy of genetic sequence analysis are achieved by the use of diverse CPU-intensive bioinformatics tools and algorithms (e.g., analysis of global similarities [3, domain and motif analysis [4, analysis of the relevant structural [5] and functional data). Running these tools on the rapidly growing data is a time-consuming process and needs high-throughput computations to get results in a timely fashion.

Modern HPC technology and infrastructures represent an emerging and expanding instrumentation, computing, information and storage platform that often allows geographically distributed resources, which are under distinct control, to be linked together in a transparent fashion [6, 7]. The power lays not only in the aggregate computing ability, data storage, and network bandwidth that can readily be brought to bear on a particular problem, but also on its ease of use. After research efforts in nearly two decades, computing infrastructures such as the grids and clouds have matured and been widely applied to computation-intensive applications, optimized storage of large-scale distributed data, and the intelligent use of these data [8, 9, 10]. 
UConn Bio-Grid Initiatives. The UConn Bio-Grid Initiatives aim at advancing the application of modern information technology and infrastructure to various life-science disciplines. The research activities center on the development and deployment of modern Grid and Cloud technologies toward an infrastructure for automated life-science information integration, extraction and analysis [11, 12, 13]. The computing infrastructure is based on a campus-wide computational and data Grid, an effort initiated in 2004. This infrastructure is supporting several collaborative, interdisciplinary research projects, led by our project staff from Schools of Engineering and Medicine. Also an ongoing effort is the development of general-purpose middleware support for the transfer of sensitive data. Educational programs associated with the Bio-Grid Initiatives include the development of new and re-development of current courses to incorporate inventions coming out of the research, a new cross-disciplinary bio-computing minor, as well as new degree tracks emphasizing the application of High-Performance Computing to different life-science disciplines. An annual scientific meeting, the International Bio-Grid Workshop has been held in conjunction with the research and educational activities since 2003 and has been serving as a major scientific venue for our research community.

Project Goals. This Bio-Grid REU Site is a 10-week summer site supporting ten undergrad students annually. Student participants are expected to have a sufficient background in computing or biological science and be interested in HPC. A series of training seminars, conducted by the project staff, is designed to equip students with preliminary background knowledge. The seminars introduce to students concepts about modern grid and cloud computing infrastructure and related information technology. Ongoing life-science research projects deploying the campus grid are presented to the students. The seminar series also include talks on research projects that are at the planning stage of being grid-enabled.

The Bio-Grid Initiatives are enriched in research, outreach and educational activities. Associated faculty members are enthusiastic about the REU Site to continue offering the opportunity for nation-wide undergraduate students to in- 
volve in the various interdisciplinary activities. In addition to the faculty members, graduate students currently on the collaborative projects also participate in mentoring.

The REU goals with associated objectives, including learning outcomes for students are as follows.

GOAL 1: Promote an interest in the interdisciplinary research work associated with the Bio-Grid initiatives by targeting NSF-targeted student participants underrepresented minorities, students with disabilities, women, and veterans from primarily NSF-targeted institutions in the early stages of their academic careers. Anticipated results/objectives follow.

1.1 Identify, recruit and receive at least $25 \%$ applications from NSF targeted students.

1.2 REU program will be made up of at least 30\% NSF-targeted students.

$1.370 \%$ of REU students will be from targeted institutions (nonPhD-granting institutions).

$1.4100 \%$ of REU students will have completed sophomore or junior year of college.

1.5 Program will be made up of $50 \%$ life science students and $50 \%$

CSE students with an interest or course experience in HPC.

GOAL 2: Promote the acquisition of skills allowing computer scientists and biologists to function in an interdisciplinary working/research environment. Anticipated results/objectives follow.

2.1 Software engineering students will be introduced to methods to formalize and solve problems emerging from life-science disciplines. 2.2 Life-science students will be introduced to information processing techniques.

2.3 Students will value the importance of interdisciplinary knowledge. 
2.4 Students will be able to apply the scientific method in hypothesisdriven research.

2.5 Students will be able to engage in self-directed research.

2.6 Students will be able to communicate research results in verbal and written form.

2.7 Students will practice taking responsibility as members of a research team.

2.8 Students will value the importance of teamwork in scientific research.

GOAL 3: Attract NSF-targeted students to become researchers and professionals in their specialized discipline (computer science/biological sciences) but with the ability for interdisciplinary work. Anticipated results/objectives follow.

3.1 Students will be able to make informed decisions about a career in science/engineering after discovering if such a life appeals to them. 3.2 Students who so desire will make the successful transition to appropriate grad programs.

\section{Program Structure and Associated Activities}

The REU Site actively incorporates feedback from students and mentors while structuring the program. Social activities such as cook-outs, pizza parties and baseball games are jointly planned with other REU programs hosted at UConn and are scheduled at the organizational meeting before program starts. In general, the program time lines are as follows:

Day 1: Orientation, Campus Tour, Program Overview, Lunch with Faculty, 115 Ice Cream Social

Week 1: Technical Seminars (mornings); Responsible Conduct of Research Seminars (afternoons)

Week 2: Lab Tours, Center (BiBCI: Bioinformatics and Biocomputing Institute) Tours 
Week 3: Invited HPC Talks from BECAT (Booth Engineering Center for Advanced Technologies)

Week 4: Seminar on Proposal Writing, targeting NSF Graduate Research Fellowships

Week 5: Remote Bio-Grid Workshop (may be in conjunction with the student Mini-Symposium)

Week 6: Student Proposal Presentations

Week 7: Seminar on Grad School Application

Week 8: Seminar on Technical Writing and Presentations

Week 9: Diversity Forum: Challenges and Choices in Higher Education (Panel Discussions)

Week 10: Bio-Grid Symposium (Final Project Presentations)

A major component of the program is for students to actively involve in one research project. After the training seminars in the first week, students discuss 135 with the project staff about their interests and a research supervisor is assigned to each student. Under the supervision of the project staff, students have their lab space and closely interact with their supervisor and graduate students currently on the project. Project supervisors design a component of the assigned project for students to work on. Mismatches between student and mentor are resolved early in the program, allowing these students to successfully complete the project. REU students also have the opportunity to remotely attend the annual International Bio-Grid Workshop. Faculty members associated with the Bio-Grid Initiatives are all welcome to propose student projects.

Example Project: Basic Grid/Cloud Infrastructure. The development of the 145 Bio-Grid portal focuses on establishing an extensible and robust Application Programming Interface (API), based on standard procedures many life-science applications require when executing in a grid environment 14, 15, 16, 17, 18, 19, 20, 21]. This involves various compute platforms, using several different native queue managers (e.g., OpenPBS, Condor, fork), a variety of operating systems 

connections (e.g., GigE, Fast Ethernet, T1) on campus.

The Bio-Grid is based on the Globus Toolkit middleware version 2.2.4 and the web portal is served by Apache HTTP Server version 2.0. (All of the web portal pages are dynamically created using the PHP hypertext preprocessor web portal page also allows the strict use of security and authentication procedures, defining a fine grained custom interface for each grid user. Several grid user Access Control Levels (ACLs) are defined for un-authenticated, general, system administrator, and grid administrator web portal page access. The base Globus Toolkit middleware Meta computing Directory Service (MDS) information is stored in the Bio-Grid database and can be queried and displayed directly. The job monitoring system are designed to be an extremely lightweight and nonintrusive tool for monitoring applications and resources on the Bio-Grid.

Example Project: HPC Middleware Development. For a grid or cloud to serve 165 as the infrastructure for on-demand integration of sensitive clinical practice and health-care information, additional data management and replication mechanisms should be provided, as the current middleware mainly deals with flat files but not to the metadata level. This project concentrates on creating a Bio Data Management unit (BDM unit) that interfaces with the middleware and provides additional metadata management capabilities. For example, medical images are stored in DICOM servers [22, 23, 24] in the hospital. Each image may consist of one or several DICOM files representing portions of the imaged body. The first role of the BDM unit is therefore to serve as an interface between the grid Storage Element and the DICOM server by assigning a logical file name to each image. For each new DICOM image generated by an image producer, a logical file name is created and registered into the replication system. There is not necessarily a physical file instance behind this logical file number but rather a virtual file consisting of a set of DICOM files, that can be reconstructed on the fly by the BDM unit if a request for this logic file number comes in. For 

functions of a small percentage of the proteins in these genes. Often protein functions ascribe to some recurring sub-structural motifs (or minimotifs, as they often contain less than 15 amino acids), which usually present specific positional 
network is a key component of the Unified Medical Language System (UMLS) project initiated in 1986 by the U.S. National Library of Medicine (NLM). The goal of the UMLS is to facilitate associative retrieval and integration of biomedical information so researchers and health professionals can use such information 
distributed semantic network system, based on a task-based and message-driven model to exploit both task and data parallelism while processing queries.

Bio-Grid Workshop Participation. We initiated in 2003 the Annual Bio-Grid Workshop (International Workshop on Biomedical Computations on the Grid). of the possibilities and advantages linked to the development, deployment and evaluation of Grid technologies in broadly biology-related research and practice. Here the context of "biology" covers the whole range of information from molecular level (genetic and proteomic information) through cells and tissues, to the individual (clinical practice) and the population level (social healthcare). Since its initiation in 2003, BioGrid events have usually been held world wide in June or July in such cities as Tokyo, Chicago, Cardiff (UK), Singapore, Rio de Janeiro, and Berlin (Germany), all featuring invited speeches, a technical program and plenary sessions. In 2009 we were able to work with the European 255 Health-Grid Association to design a Berlin-Storrs joint session of the Bio-Grid program and had our summer REU students remotely attend this session.

\section{The Research Environment}

The project investigators all have extensive experience in undergraduate teaching, research supervision, and curriculum development. The REU staff and faculty affiliated with Bio-Grid Initiatives have long collaborated on interdisciplinary research projects and have been involving undergraduate students in the research work.

The CSE faculty represents a wide diversity of research disciplines and are recipients of major research grants from federal agencies such as the NSF, NIH, DARPA, ONR, DoEd, etc. The twenty-eight faculty members came from eleven different countries, representing another dimension of diversity. The department has a long tradition of being at the forefront of computing education, offering 
broad based educational programs in computer Science and Engineering with bachelor, master and doctoral degrees.

The university has a 104-node HPC Cluster and a 64-node SGI Altix 3700 available for summer students. The 104-node (1,408-core) HPC cluster contains both CPU and GPU resources, enabling a massive amount of computing power. There are 3 classes of nodes available in the cluster: (1) 60 Intel Westmere compute nodes, each node with 12 Intel Xeon X5650 Westmere cores (total of 275720 cores) and 48 GB of RAM. (2) 40 Intel Sandy Bridge compute nodes, each node with 16 Intel Xeon E5-2650 Sandy Bridge cores (total of 640 cores) and 64 GB of RAM; and (3) 4 NVIDIA GPU nodes, each node with 8 NVIDIA Tesla M2050 GPUs, 12 Intel Xeon X5650 Westmere cores and 48 GB of RAM. The nodes are configured to use LSF job scheduler, and uses uses low-latency Infiniband network interconnect. 200 TB of high-performance GPFS storage is made available to the cluster. The SGI Altix system consists of (1) an SGI Altix 350 system with 8 Intel Itanium 2 Processors (1.5GHz with $6 \mathrm{MB}$ cache), each with 8 GB Memory, and an 80 GB SATA Hard Drive as the front-end server; and (2) an SGI Altix 3700 Bx2 system with 64 Intel Itanium 2 Processors (1.5GHz with $4 \mathrm{MB}$ cache), each with 64 GB memory and 146 GB 10K RPM SCSI Hard Drive, with additional four 300 GB 10K RPM Ultra320 SCSI Hard Drives. These twin systems are networked to the School's existing SGI Onyx-4 Visualization System to provide a complete compute-to-visualization package for project staff and summer students.

\section{Student Recruitment and Selection}

In addition to a dedicated REU website linked from the NSF, we encourage student applicants from across the nation by extensively sending out brochures and invitations. Students from institutes where less research opportunities are available are especially encouraged to apply. We have previously collaborated with investigators from minority institutions on NSF funded research and educational projects. UConn has a rich history of working closely with minority 
institutions. We work closely with the university to publicize the REU site among those traditionally minority institution $\mathbb{1}^{1}$ and among student members of the National Society of Black Engineers, the Society for Hispanic Professional dent study session for credits with the faculty supervisor whose project they feel interested in.

We work with the Office of Veterans Affairs and Military Programs at UConn to actively recruit veteran students via the National Association of Veterans' 325 Programs Administrators (NAVPA). NAVPA's member institutions include such

\footnotetext{
${ }^{1}$ United States Department of Education Accredited Postsecondary Minority Institutions.
} 
NSF targets as minority-serving community colleges and other institutions lacking research opportunities. The primary role of the office at the university is to provide direct support for student veterans as well as to create a universitywide support network for veterans throughout the university. Additionally, the Division of Student Affairs at UConn owns a house that serves as a drop in center for student veterans. This house has been available to REU student veterans. The office also assists in accessing student veterans across the nation for recruitment.

We work with the Center for Students with Disabilities (CSD) at UConn to actively recruit disabled students from UConn and other institutions to participate in the summer REU. UConn's CSD has seen the rapid growth in the number of students with disabilities accessing higher education. In 1993, the University served approximately two hundred students with disabilities. In 2008-2009, the CSD worked with more than one thousand. The CSD program has been recognized as a "model program" by the Association on Higher Education and Disability (AHEAD). The CSD has an extensive peer mentor network where entering students with disabilities are paired with current students to assist with their transition to the university community. The service is extended to summer REU students with disabilities. The CSD is also committed to offering 345 specialized training required for mentors to accommodate REU students.

The application requires (1) personal information including prior research, project and any course experiences in HPC; (2) a brief research statement about personal and professional goals and how the REU experience would further these goals; (3) the selection and prioritization of three potential research topics; and (4) provide two letters of reference, at least one of which should be from the applicant's institution.

Our recruitment goals are to attract a group of highly motivated students, roughly half majoring in computer science and half majoring in a biology-related field, with appropriate academic records and a targeted percentage from non$355 \mathrm{PhD}$ granting institutions. However, due to the partnerships we have established with former participants' schools and other institutions, we have been using our 
contacts at each to help select promising students whose academic background may miss the bar yet show high potential in non-GPA ways. Participating faculty of the REU project performs preliminary evaluations of applications, based on academic performance, research statement, and letters of reference. Final decisions are based on the student's (a) academic background and perceived interest and enthusiasm for the interdisciplinary research, (b) home institution (non-PhD or limited research given preference), (c) ethnic and gender diversity, if available (applicant's gender, race and ethnic background are requested on an optional basis), and (d) disability and veteran status. A modified rolling admission procedure is employed until all slots are filled. Mentors and students are matched based on mutual preferences.

\section{Program Evaluation and Reporting}

Evaluation of the student experience commences with an initial survey in which REU students articulate their goals for the program. Formative evaluation, i.e., continued evaluation with the possibility of program modification as indicated, is conducted during the first two-three weeks and at the mid-point in addition to summative evaluation (end-of-program surveys for both students and mentors, and follow-up communication with students). The project applies both qualitative and quantitative evaluation methods to assess the goals, as well as the outcome-based objectives for each goal. Assessment is aimed at measuring objectives/outcomes associated with students' acquisition of research skills, especially in the appropriate cross-discipline, gaining an understanding of life in the research lab, and eventual enrollment in appropriate graduate programs. 380 To assess success of the project in recruiting diverse REU students, we track all applications, including demographic data that is supplied by the applicant. We determine what level of recruitment (personal letter, phone call, recruitment visit, etc.) provides the best return - both in attracting applicants and in attracting participants - for our recruitment effort. Both qualitative (individual and group interview) and quantitative (survey) approaches are used to assess 
student experiences and outcomes, and focus on the specific objectives.

Upon arrival, REU students are given a questionnaire on their goals and expectations. Each lab is visited at least once/week during the first month of the program to informally monitor progress as part of ongoing formative evaluation. Interview questions and observations are aimed at determining how well the REU student "fits" in the lab, including the level of interaction with other lab personnel and the student's perceptions regarding how $\mathrm{s} /$ he is progressing on a project. We plan a formal mid-program evaluation, possibly in the context of a group breakfast or lunch meeting. Mentors (REU faculty) are also surveyed (a) within the first few weeks; (b) at midpoint and (c) at the end of the program to determine their perceptions of each student's progress. Students' ability to communicate results are assessed both by mentor's observations and by the final presentation which culminate the summer REU experience. At the end of the summer, REU students are given comprehensive survey questionnaires designed to elicit feedback on all aspects of the program, from housing and social activities to a detailed evaluation of their research experience and their intellectual experiences in the form of seminars and ancillary activities. This mixed method designed for assessment can, especially after the program is in place for a few years, provide data to assess the efficacy of our REU site at promoting increased representation of underrepresented minorities, veterans and persons with disabilities in research.

Maintaining Contact. Post-program contact with the students has been facilitated by the mentor-student relationships developed over the summer program, in addition to utilizing traditional phone numbers and email addresses. We encouraged students to take home their posters from the end-of-summer conference, and contacted a faculty member at each student's home institution to let them know about the poster and encourage them to have the REU student give a talk. Students were contacted by email periodically during the following semesters and polled as to their plans and career choices. We also solicited the first year REU students as ambassadors and asked them to encourage future 
applicants from their home colleges.

\section{Project Outcomes}

Our Goal (1) was to "promote an interest in the interdisciplinary research work associated with the Bio-Grid initiatives by targeting NSF-targeted student participants - underrepresented minorities, students with disabilities, women, and veterans from primarily NSF-targeted institutions in the early stages of their academic careers", with an objective of anticipating half participants with an interest or background in HPC and another half with a biology-related background. Tables 1 and 2 summarize our results about participants, offers made and applicants. "Target Schools" refers to Non-PhD granting institutions. Our Goal (1) was met. The objective of recruiting half CS students and half Biorelated students was being approached, with an improvement from 8:2 (CS:Bio) in 2008 to 6:4 (CS:Bio) in 2010. The ratio slightly dropped in 2012 to 4:2 (CS:Bio) and in 2013 to 7:2 (CS:Bio). We believe this drop has to do with the rather small cohort funded in 2012 and 2013 (15 total). Note that the classification of students into CS or BIO was simply to assist in making offers and organizing student teams. The classification was more based on experience, not on student's major. A CS student is one who is experienced in computing, especially HPC. A BIO student is one who had prior exposure to biology courses or wet-lab experience. For example, a Mechanical Engineering major with significant HPC experience is put in the CS group. A Computer Science major with a Bioinformatics concentration and relevant wet-lab experience is regarded as a BIO student.

Our Goal (3) was to "attract NSF-targeted students to become researchers and professionals in their specialized discipline (computer science/biological sciences) but with the ability for interdisciplinary work". In this regard, the percentage of female participants has risen from $10 \%(1 / 10)$ in 08 to $60 \%$ in 2010 , with a drop to $33 \%(2 / 6)$ in 2012 and $22 \%$ in $2013(2 / 9)$. While there is certainly room for improvement in this outcome, we did observe that more than 
Table 1: Bio-Grid REU Site Statistics 2008-2010: Participants/Offers Made/Applicants

\begin{tabular}{|c|c|c|c|c|c|c|}
\hline Year & Total & $\begin{array}{l}\# \quad \mathrm{Fe}- \\
\text { male }\end{array}$ & $\begin{array}{l}\# \\
\text { Under- } \\
\text { repre- } \\
\text { sented } \\
\text { Minori- } \\
\text { ties }\end{array}$ & $\begin{array}{l}\text { \# from } \\
\text { Target } \\
\text { Schools }\end{array}$ & $\begin{array}{l}\# \quad \text { of } \\
\text { CS- } \\
\text { related } \\
\text { Major }\end{array}$ & $\begin{array}{l}\# \quad \text { of } \\
\text { Bio- } \\
\text { related } \\
\text { Major }\end{array}$ \\
\hline 2008 & $10 / 14 / 38$ & $1 / 4 / 9$ & $1 / 4 / 7$ & $4 / 7 / 18$ & $8 / 12 / 31$ & $2 / 2 / 7$ \\
\hline 2009 & $10 / 17 / 54$ & $3 / 6 / 19$ & $1 / 4 / 8$ & $2 / 6 / 20$ & $7 / 10 / 40$ & $3 / 4 / 14$ \\
\hline 2010 & $10 / 15 / 68$ & $6 / 8 / 22$ & $2 / 6 / 12$ & $5 / 9 / 37$ & $6 / 10 / 41$ & $4 / 5 / 27$ \\
\hline Total & $30 / 46 / 160$ & $10 / 18 / 50$ & $4 / 14 / 27$ & $11 / 22 / 75$ & $21 / 32 / 112$ & $9 / 11 / 48$ \\
\hline
\end{tabular}

from non-PhD granting institutions rises from 40\% (4/10) in 2008 to $67 \%(6 / 9)$ in 2013. In addition, we have been continuing our efforts in recruiting students that are under-represented minorities (Black, Hispanic, Native American, Native Hawaiian). In 2013, 6 offers were made to underrepresented minorities,

while 4 such offers were made in each of 08 and 09, and 3 in 12. Although not all offers to under-represented minorities were taken, we did observe a significant increase of minority applicants, especially from institutes we've targeted. The lower yield rate from female and minority applicants is likely due to the fact that the federally sponsored REU programs specifically encourage diversity and broadening participation, and qualified female and minority applicants are likely to receive more offers to choose from. Finally, we made two offers in 2013 to two applicants who indicated disability. A student who was diagnosed with Crohn's Disease joined us for the summer of 2013. We worked with the housing department and the Center for Students with Disabilities at UConn to 460

Bio-Grid students were recruited from universities as follows.

- Bowdoin College 
Table 2: Bio-Grid REU Site Statistics 2012-2013: Participants/Offers Made/Applicants

\begin{tabular}{|l|l|l|l|l|l|l|}
\hline Year & Total & $\begin{array}{l}\# \quad \text { Fe- } \\
\text { male }\end{array}$ & $\begin{array}{l}\# \\
\text { Under- } \\
\text { repre- } \\
\text { sented } \\
\text { Minori- } \\
\text { ties }\end{array}$ & $\begin{array}{l}\text { Target } \\
\text { Schools }\end{array}$ & $\begin{array}{l}\# \text { of } \\
\text { Major }\end{array}$ & $\begin{array}{l}\# \text { of } \\
\text { Bio (or } \\
\text { non- } \\
\text { CSE) } \\
\text { Major }\end{array}$ \\
\hline \hline 2012 & $6 / 8 / 88$ & $2 / 4 / 18$ & $1 / 3 / 9$ & $2 / 4 / 33$ & $4 / 5 / 60$ & $2 / 3 / 28$ \\
\hline 2013 & $9 / 16 / 117$ & $2 / 6 / 36$ & $1 / 6 / 18$ & $6 / 9 / 42$ & $7 / 9 / 72$ & $2 / 7 / 45$ \\
\hline \hline Total & $15 / 24 / 205$ & $4 / 10 / 54$ & $2 / 9 / 27$ & $8 / 13 / 75$ & $11 / 14 / 132$ & $4 / 10 / 73$ \\
\hline
\end{tabular}

- Carnegie Mellon University

- City University of New York - Hunter

- Colorado Mesa University

- Duke University

- East Coast Polytechnic Institute

- Fairfield University

- Hobart College

470

- Lewis-Clark State College

- Loyola Marymount University

- Manchester Community College

- Manhattanville College

- Rhode Island College

475

- Siena College

- Swarthmore College

- Tufts University

- University of California San Diego

- University of Connecticut

480

- University of Delaware

- University of Hartford 
- University of Maine, Orono

- University of Massachusetts Amherst

- University of Pennsylvania

- University of Puget Sound

- University of Tennessee at Chattanooga

- University of Texas Dallas

- Western New England College

Our Goal (2) was to "promote the acquisition of skills allowing computer scientists and biologists to function in an interdisciplinary working/research environment". Students conducted research under the supervision of mentors, interacting with graduate students and postdocs, participating in research meetings and experienced life as a researcher. Whenever possible, a Bio student and a CS student were teamed up to experience interdisciplinary teamwork. Students all reported that the REU gave them the understanding they needed to assess whether a research career is for them. Ongoing formative evaluation, consisting of meetings with students at the end of the first week as well as midexperience, allowed for timely corrections of any problems. All mentors reported that students were good team members. All students reported viewing research as largely a team effort, and gave specific examples of being able to ask questions of different grad students, or of learning different techniques from different groups. All mentors reported students made progress toward independence in research, with a majority reporting students were able to work with complete independence by the end of the program, indicating the immediate objectives of Goal (2) were met.

Although the extent to which Goals (2) and (3) are successfully accomplished can only be determined with the passage of time, the structured interviews during the course of the summer program and through follow-up interviews immediately after the completion of the program has a strong indication of the successful accomplishment of these goals. It's also noted that most students indicated there was no direct cause-effect relationship between the Bio-Grid 
summer program and their career choice, although all students acknowledged the interdisciplinary research experience and agreed it has provided valuable input in their post-program research and course activities.

Technical seminars in the first week of program invited mentors to give a research presentation (Figures 1, 2 and 3). This provided sufficient project and mentor information to students before the project assignment at the end of the first week. Departmental seminars on the graduate school application process, research proposal writing targeting the NSF graduate fellowship application, technical report writing and effective scientific presentation have also been very well received. Weekly group meetings with the project staff helped resolve any potential problems in a timely manner. Joint $R E U$ seminars were integral to our program. Among them are an Ethics workshop presentation addressing ethics in research, a stem cell ethics seminar, and a discussion regarding ethics.

${ }_{525}$ Starting 2010, the ethics workshop evolved into an RCR (Responsible Conduct of Research) seminar series (Figure 4), spanning the entire first week of program. The Diversity in the Sciences Workshop evolved from a presentation to a panel discussion, utilizing faculty and staff from across the campus (Figures 5 and 6). The mid-term Bio-Grid mini-Symposium required all Bio-Grid students to prepare a 15-min or so oral presentation, accompanied by a one-page research summary. The mini-symposium was designed to get students familiar with the "proposal defense" stage when in graduate school. It also equipped students with knowledge about grant proposal writing. The final Bio-Grid Symposium program was the culminating event. Each project was allotted 30-min for an oral presentation. A technical report was due before students left the program. Collegiality was fostered by many social events - most of them involving all summer research participants (ice cream social, barbecue, baseball game) and others just the Bio-Grid REU program (initial orientation). Summer research students were housed together in a separate section of the summer dormitory, 540 and interacted with Community Assistants (grad students working in Housing) who were connected to the summer research program. 
Outcomes of continued post-program mentoring for REU participants. We regularly contact Bio-Grid alumni and offer assistance in recommendation letters and in preparation for graduate fellowship applications for those applying to graduate school. As of April 2016, Bio-Grid alumni have won the NSF Graduate Research Fellowship in 2016 (Stephanie Kowlton, Bio-Grid 2012) and 2012 (Brittany Nkounkou, Bio-Grid 10), an Alfred P. Sloan Foundation Graduate Scholarship in 2012 (Brittany Nkounkou, Bio-Grid 10), an honorable mention of the NSF Graduate Research Fellowship in 2012 (Michael Zuba, Bio-Grid 09), and a GAANN Fellowship by US Dept. of Education in 2010 (Michael Zuba, Bio-Grid 10).

Among the 15 REU students recruited from 12-13, 8 are now in graduate school (6 PhD, $2 \mathrm{MS}$ ). 4 students are in the process applying to graduate school. By the summer of 2016 around $80 \%$ of REU students recruited from 12-13 will be pursuing a graduate degree. Among the 29 students recruited from 08-10, 13 students are attending or have finished graduate school (7 PhD, $1 \mathrm{MD} / \mathrm{PhD}$, $1 \mathrm{MD}, 4 \mathrm{MS}$ ). 6 students currently in industry are applying to graduate school and expect to start an $\mathrm{MS} / \mathrm{PhD}$ program within a year. One student is applying to medical school. In summary, by next summer around $70 \%$ of REU students recruited from 08-10 will have completed (or be pursuing) a graduate degree.

Many of the Bio-Grid alumni have indicated their experience in HPC have helped them throughout their graduate study.

Bio-Grid alumni entered such graduate schools or medical schools as

- Boston University

565

- Columbia University

- Cornell University

- Rensselaer Polytechnic Institute

- Stanford University

- University of California, Santa Barbara

570

- University of Connecticut (grad school and medical school)

- University of Maryland, College Park 
- University of North Carolina Chapel Hill

- University of Pennsylvania

- University of Pittsburgh (Medicine)

- University of Texas (Medicine)

Additional student outcomes include peer-reviewed scientific publications in both HPC and bioinformatics co-authored with summer REU students in major journals and conferences [41, 42, 43, 44, 45, 46, 47, 48, 49.

Outcome of Faculty From REU Student Mentoring. The outcome of the REU program also involves the benefits faculty and the CSE department would gain from the investment of time and resources in mentoring REU students. Experience gained from our REU Site program concerning assessment and recruitment was used extensively in a few current and pending grants by colleagues. Throughout the funding periods, twelve faculty members and fifteen graduate students have mentored summer students.

\section{Lessons Learned: Incorporating HPC in an Interdisciplinary REU}

This section discusses the experience gained and lessons learned from the perspectives of an REU Site designed to incorporate High-Performance Computing in interdisciplinary research and education. Recruiting Students with Sufficient Exposure to HPC. Concepts of HPC arises from every aspect of parallelism in computing, which is relevant to most of the courses offered in a typical undergraduate Computer Science or Computer Engineering curriculum. Ideally participants on the computing side should have finished such courses as Operating Systems, Computer Architecture and Algorithm Analysis; have some experience in parallel programming; and are interested in life-science related research work.

Recruiting Students in Biology-Related Majors. Students on the BIO side may be in such majors as Molecular Biology, Evolutionary Biology, Neuroscience, 
Biomedical Engineering, etc.; preferably have finished one year of courses in participant in 2010, who did not have a plan to do a $\mathrm{PhD}$ when admitted to 
Bio-Grid, later published a paper in 2011, won two graduate research fellowships as a college senior in 2012 and as of now has been a CS PhD student

630 (Cornell) and has been serving as President of Black Graduate and Professional Student Association. One of the student's mentors was our Cloud Computing GAANN (Graduate Assistance in Areas of National Need) doctoral fellow, an underrepresented minority student as well.

\section{Acknowledgement}

635 Funding. The Bio-Grid REU Site acknowledged funding by the National Science Foundation in grants CCF-0755373 (2008-2010) and OCI-1156837 (2012-2014).

Faculty mentors. Faculty mentors and consultants inculde Jun-Hong Cui (CSE), Swaminathan Hariharan (Educational Psychology), Richard Jones (Physics), Aggelos Kiayias (CSE, formerly), Kristen Kimbal (Physiology and Neurobiol${ }_{640}$ ogy), Ion Mandoiu (CSE), Thomas J. Peters (CSE), Sanguthevar Rajasekaran (CSE), Heather Read (Psychology and Biomedical Engineering), Bing Wang (CSE) and Xiaoyan Wang (Family Medicine).

Collaborating UConn REU Sites. The Bio-Grid REU Site also acknowledged the joint events co-hosted by collaborating REU Sites at UConn by the Depart${ }_{645}$ ments of Chemical Engineering, Chemistry, Electrical Engineering, as well as Physiology and Neurobiology.

\section{References}

[1] S. K. Prasad, A. Y. Chtchelkanova, S. K. Das, F. Dehne, M. G. Gouda, A. Gupta, J. Jaja, K. Kant, A. La Salle, R. LeBlanc, et al., Nsf/ieee-tcpp curriculum initiative on parallel and distributed computing: core topics for undergraduates, in: SIGCSE, Vol. 11, 2011, pp. 617-618.

[2] K. Sayood, Introduction to data compression, Newnes, 2012. 
[3] W. Pearson, Using the FASTA program to search protein and DNA sequence databases, Methods Mol. Biol. 24 (1994) 307-331.

[4] A. Bateman, et al., The Pfam protein families database, Nucleic Acids Res. 30 (2002) 276-280.

[5] L. LoConte, S. Brenner, T. Hubbard, C. Chothia, A. Murzin, SCOP database in 2002: refinements accommodate structural genomics, Nucleic Acids Res. 30 (2002) 264-267.

[6] F. Berman, G. Fox, A. J. Hey, Grid computing: making the global infrastructure a reality, Vol. 2, John Wiley and sons, 2003.

[7] I. Foster, C. Kesselman, The Grid: Blueprint for a New Computing Infrastructure, Morgan Kaufmann, San Francisco, 1999.

[8] R. Butler, D. Engert, I. Foster, C. Kesselman, S. Tuecke, J. Volmer, V. Welch, A National-Scale Authentication Infrastructure, IEEE Transactions on Computer 33 (12) (2000) 60-66.

[9] M. L. Green, R. Miller, Molecular Structure Determination on a Computational and Data Grid, in: Proceedings 4-th IEEE/ACM Symposium on Cluter Computing and the Grid - BioGrid Workshop, CD-ROM, 2004.

[10] I. Foster, The Grid: A New Infrastructure for 21st Century, Physics Today 55 (2) (2002) 42-47.

[11] C.-H. Huang, Bio-Grid: A Collaborative Environment for Life-Science Research, in: Proceedings of the 20-th International Symposium on Critical Care and Medicine, 2005, pp. 123-132.

675 [12] C.-H. Huang, Bio-Grid: Bridging Life Science and Information Technology, in: Proceedings of the 5-th IEEE/ACM Symposium on Cluster Computing and the Grid (BioGrid Workshop), CD-ROM, 2005.

[13] C.-H. Huang, S. Rajasekaran, High-Performance Parallel Biocomputing, Parallel Computing Journal 30 (9-10) (2004) 999-1000. 
[20] C.-W. Lee, C.-H. Huang, Toward Cooperative Genomic Knowledge Inference, Parallel Computing Journal 30 (9-10) (2004) 1127-1135.

[21] Z.-Z. Chen, X. He, C.-H. Huang, Finding double euler trails of planar graphs in linear time, SIAM Journal on Computing 31 (4) (2002) 1255705

[22] D. L. Collins, J. Montagnat, A. P. Zijdenbos, A. C. Evans, D. L. Arnold, Automated estimation of brain volume in multiple sclerosis with biccr, in: 
Biennial International Conference on Information Processing in Medical Imaging, Springer, 2001, pp. 141-147.

[23] G. Comi, M. Philippi, V. Martinelli, G. Sirabian, A. Visciani, A. Cambi, S. Mammi, M. Rovaris, M. Canal, Brain Magnetic Resonance Imaging Correlates of Cognitive Impairment in Multiple Sclerosis, Journal of Neurological Science 115 (1993) 66-73.

[24] N. Losseff, L. Wang, H. Lai, D. Yoo, M. Gawne-Caine, W. McDonald, D. Miller, A. Thomas, Progressive Cerebral Atrophy in Multiple Sclerosis: A serial MRI study, Brain 119 (6) (1996) 2009-2019.

[25] S. Henikoff, J. G. Henikoff, Amino acid Substitution Matrices From Protein Blocks, in: Proceedings of Natl. Acad. Sci., 89, 1992, pp. 10915-10919.

[26] E. Birney, Hidden Markov Models in Biological Sequence Analysis, IBM J. RES. \& DEV 45(3/4) (2001) 449-454.

[27] H. M. Martinez, An Efficient Method for Finding Repeats in Molecular Sequences, Nucleic Acids Research 11(13) (1983) 4629-4634.

[28] G. Bejerano, G. Yona, Modeling Protein Families Using Probabilistic Suffix Trees, in: Proceedings of Third International Conference on Computational Molecular Biology (RECOMB), 1999, pp. 15-24.

[29] A. Apostolico, G. Bejerano, Optimal Amnesic Probabilistic Automata or How to Learn and Classify Proteins in Linear Time and Space, in: Proceedings of Fourth International Conference on Computational Molecular Biology (RECOMB), 2000, pp. 25-32.

[30] R. L. Tatusov, Altschul, S. F., E. V. Koonin, Detection of Conserved Segments in Proteins: Iterative Scanning of Sequence Databases with Alignment Block, in: Proceedings of Natl. Acad. Sci., 91, 1994, pp. 12091-12095.

[31] S. Balla, V. Thapar, S. Verma, T. Luong, T. Faghri, C.-H. Huang, S. Rajasekaran, J. del Campo, J. Shinn, W. Mohler, M. Maciejewski, M. Gryk, 
B. Piccirillo, S. Schiller, M. Schiller, Minimotif Miner: A New Tool for Investigating Protein Function, Nature Methods 3 (3) (2005) 1-3.

[32] S. Rajasekaran, S. Balla, C.-H. Huang, V. Thapar, M. Gryk, M. Maciejewski, M. Schiller, High-performance exact algorithms for motif search, Journal of clinical monitoring and computing 19 (4-5) (2005) 319-328.

[33] S. Rajasekaran, C.-H. Huang, A Randomized Algorithm for Distance Matrix Calculations in Multiple Sequence Alignment, in: Proceedings of First Knowledge Explorration in Life Science Informatics (Kelsi), LNAI 3303, Springer-Verlag, 2004, pp. 33-45.

[34] C.-W. Lee, C.-H. Huang, S. Rajasekaran, TROJAN: A Scalable Parallel Semantic Network System, in: Proceedings of the 15th IEEE International Conference on Tools eith Artificial Intelligence, 2003, pp. 219-223.

[35] M. Surdeanu, D. I. Moldovan, S. M. Harabagiu, Performance Analysis of a Distributed Question/Answering System, IEEE Trans. on Parallel and Distributed Systems 13 (6) (2002) 579-596.

[36] M. P. Evett, J. A. Hendler, L. Spector, Parallel Knowledge Representation on the Connection Machine, Journal of Parallel and Distributed Computing 22 (1991) 168-184.

[37] K. Stoffel, J. Hendler, J. Saltz, B. Anderson, Parka on MIMDSupercomputers, Tech. Rep. CS-TR-3672, Computer Science Dept., UM Institute for Advanced Computer Studies, University of Maryland, College Park (1996).

[38] C. Lee, C.-H. Huang, et al., Lasagna-search: an integrated web tool for transcription factor binding site search and visualization, Biotechniques 54 (3) (2013) 141-153.

[39] D. Lindberg, B. Humphreys, A. McCray, The Unified Medical Language System, Methods Inf. Med. 32 (4) (1993) 281-291. 
[40] A. McCray, S. Srinivasan, A. Browne, Lexical Methods for Managing Variation in Biomedical Terminologies, in: Proceedings Annual Symposium Compu. Appl. Med. Care, 1994, pp. 235-239.

[41] N. T. L. Tran, S. Mohan, Z. Xu, C.-H. Huang, Current innovations and future challenges of network motif detection, Briefings in Bioinformatics 16 (3) (2015) 497-525.

[42] N. T. L. Tran, L. DeLuccia, A. F. McDonald, C.-H. Huang, Crossdisciplinary detection and analysis of network motifs, Bioinformatics and Biology insights 9 (2015) 49.

[43] N. T. L. Tran, C.-H. Huang, A survey of motif finding web tools for detecting binding site motifs in chip-seq data, Biol Direct 9 (1) (2014) 4.

[44] C. Lee, C.-H. Huang, Searching for transcription factor binding sites in vector spaces, BMC bioinformatics 13 (1) (2012) 215.

[45] C. Lee, C.-H. Huang, Lasagna: A novel algorithm for transcription factor binding site alignment, BMC bioinformatics 14 (1) (2013) 108.

[46] C. Lee, A. Abdool, C.-H. Huang, Pca-based population structure inference with generic clustering algorithms, BMC bioinformatics 10 (Suppl 1) (2009) S73.

[47] E. Wong, B. Baur, S. Quader, C.-H. Huang, Biological network motif detection: principles and practice, Briefings in bioinformatics 13 (2) (2012) 202-215.

[48] J. Duitama, J. Kennedy, S. Dinakar, Y. Hernández, Y. Wu, I. I. Măndoiu, Linkage disequilibrium based genotype calling from low-coverage shotgun sequencing reads, BMC bioinformatics 12 (1) (2011) 1.

[49] D. Sharma, S. Balla, S. Rajasekaran, N. DiGirolamo, Degenerate primer selection algorithms, in: Computational Intelligence in Bioinformatics and 
Computational Biology, 2009. CIBCB'09. IEEE Symposium on, IEEE, 2009, pp. 155-162. 


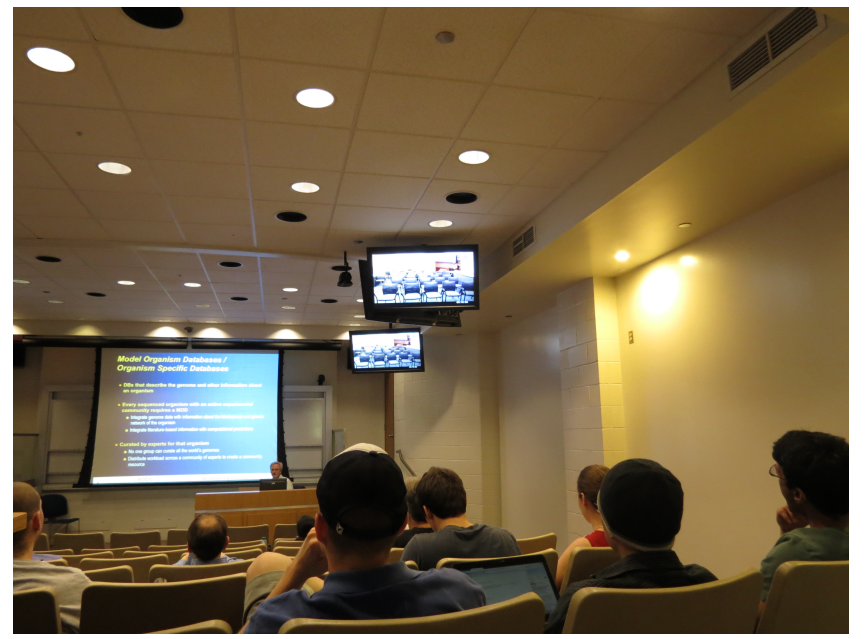

Figure 1: Technical Seminar: Model Organism Databases 


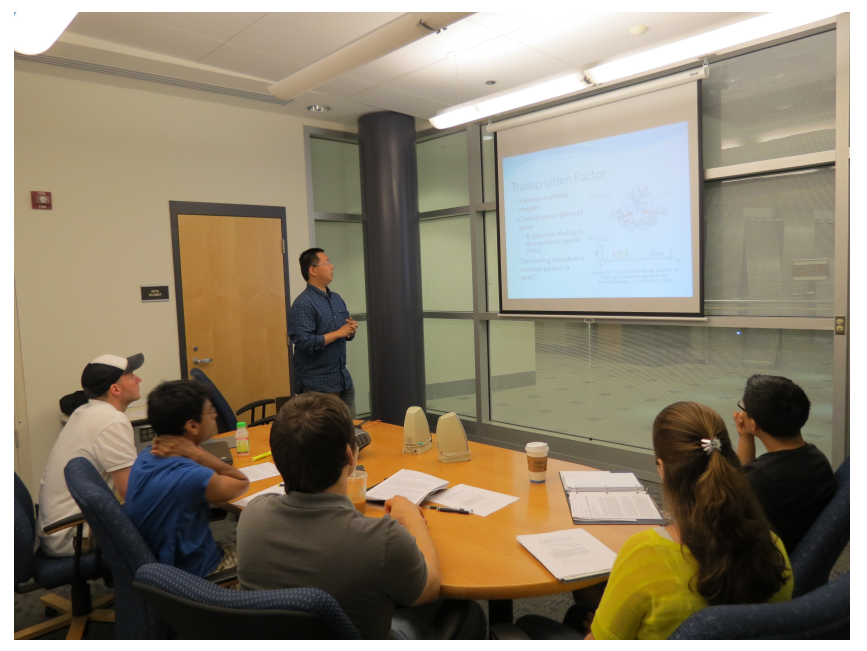

Figure 2: Technical Seminar: GPU Programming

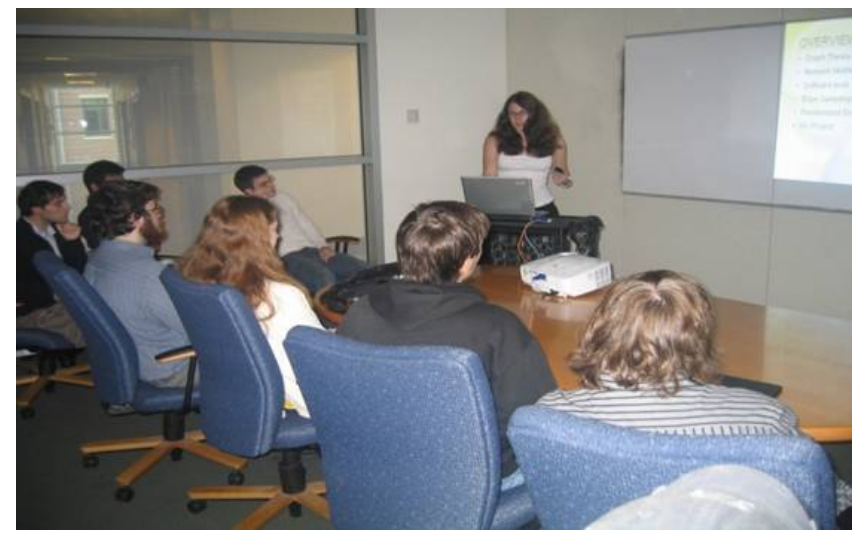

Figure 3: Technical Seminar: Storage Clouds 


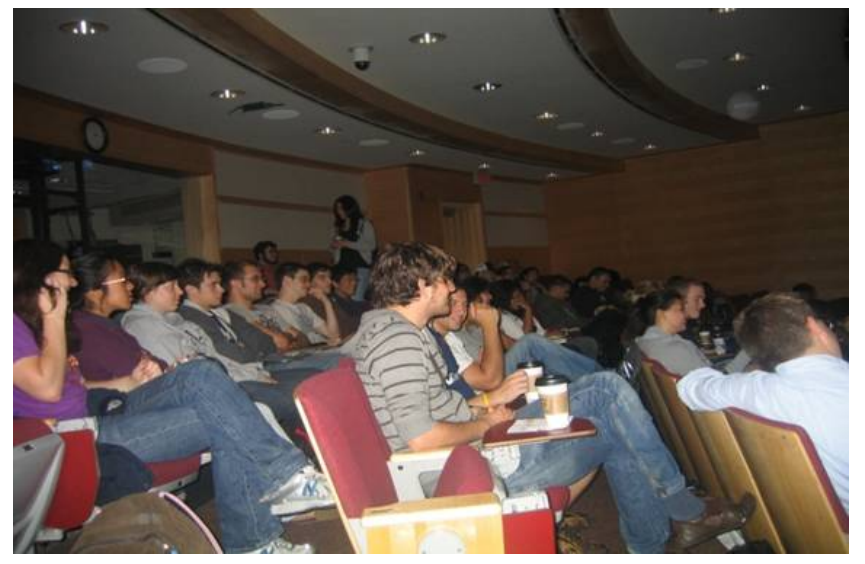

Figure 4: Responsible Conduct of Research Seminar

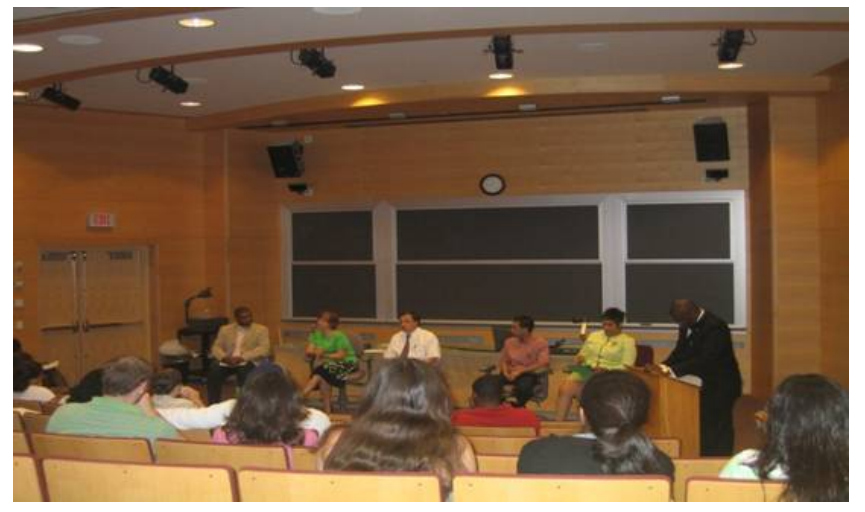

Figure 5: Diversity in the Sciences Workshop: Panel Discussions

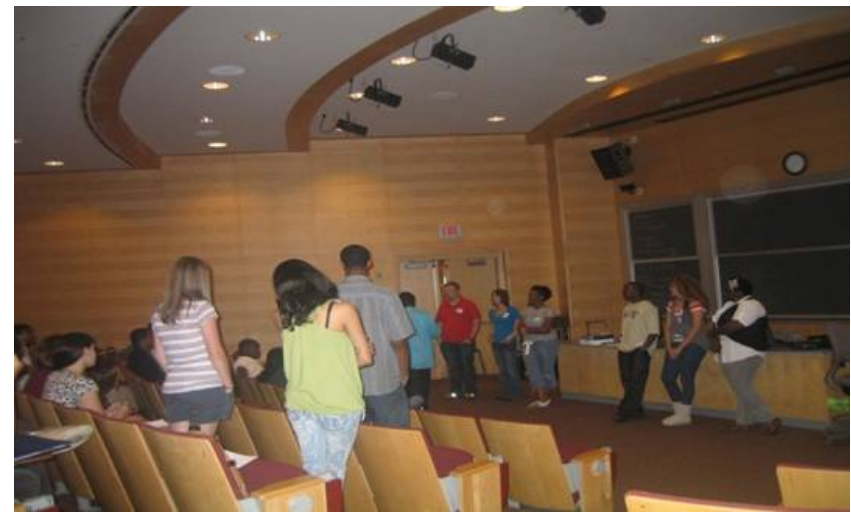

Figure 6: Diversity in the Sciences Workshop: Group Discussions 


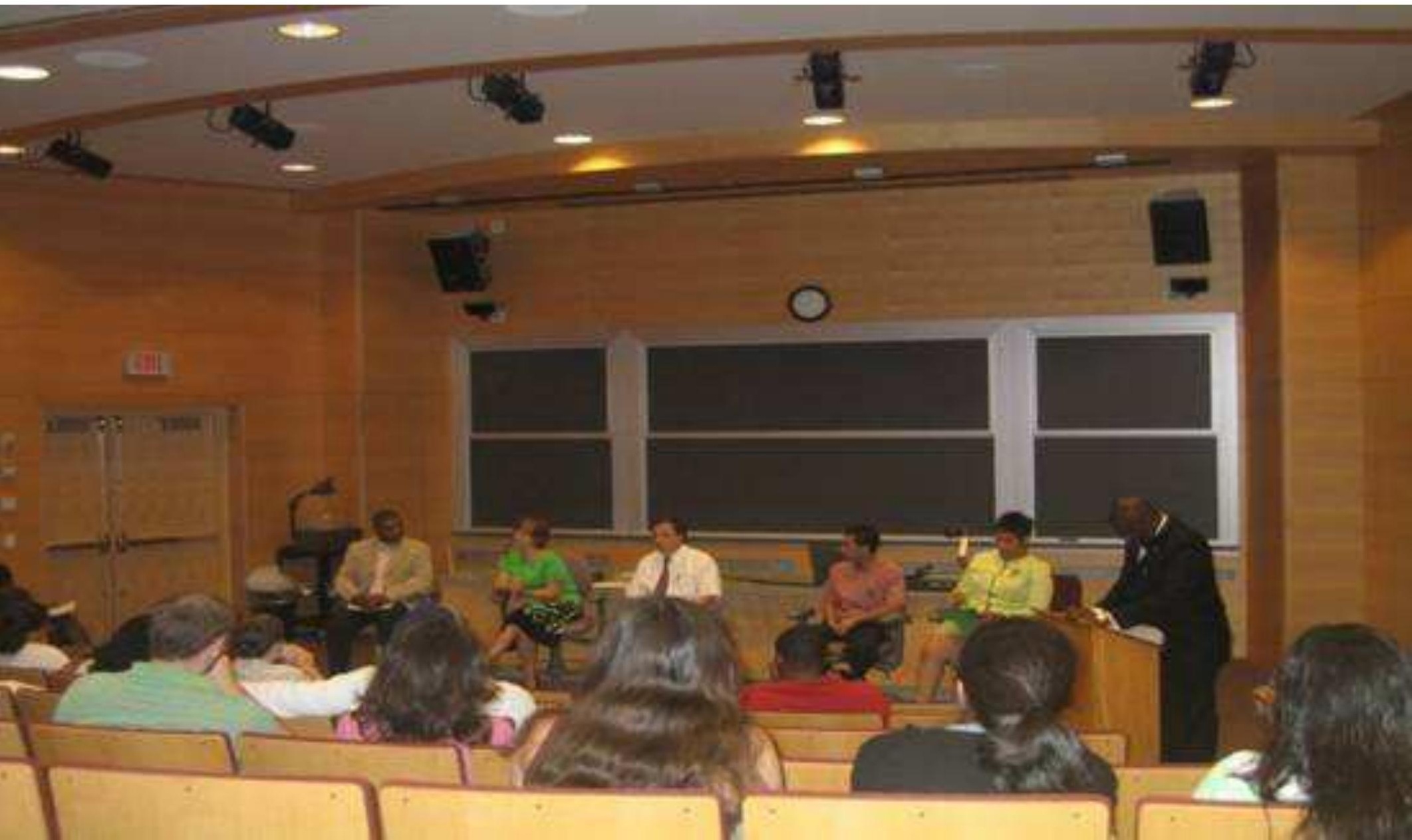




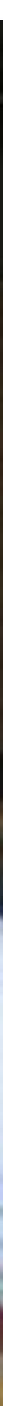




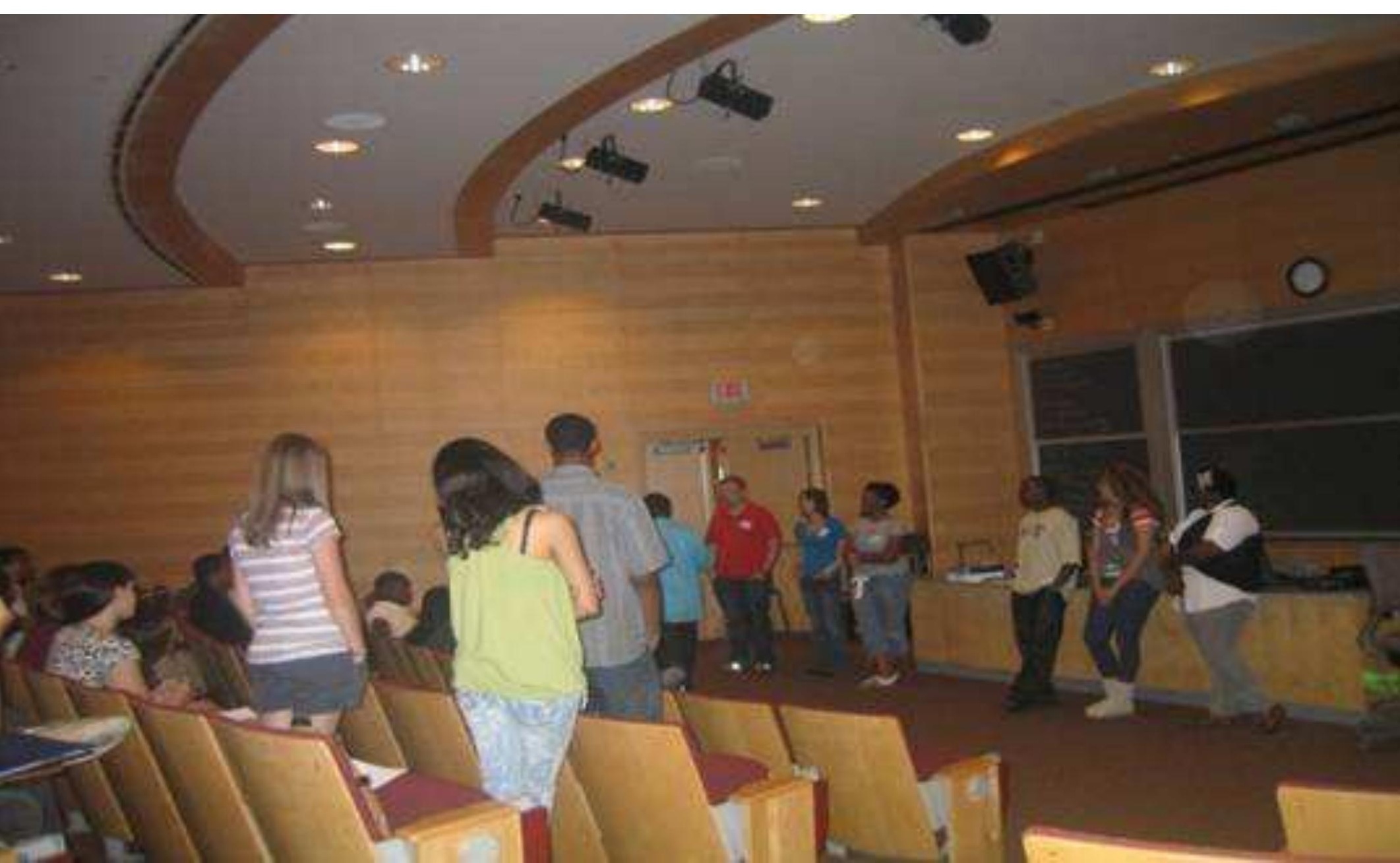




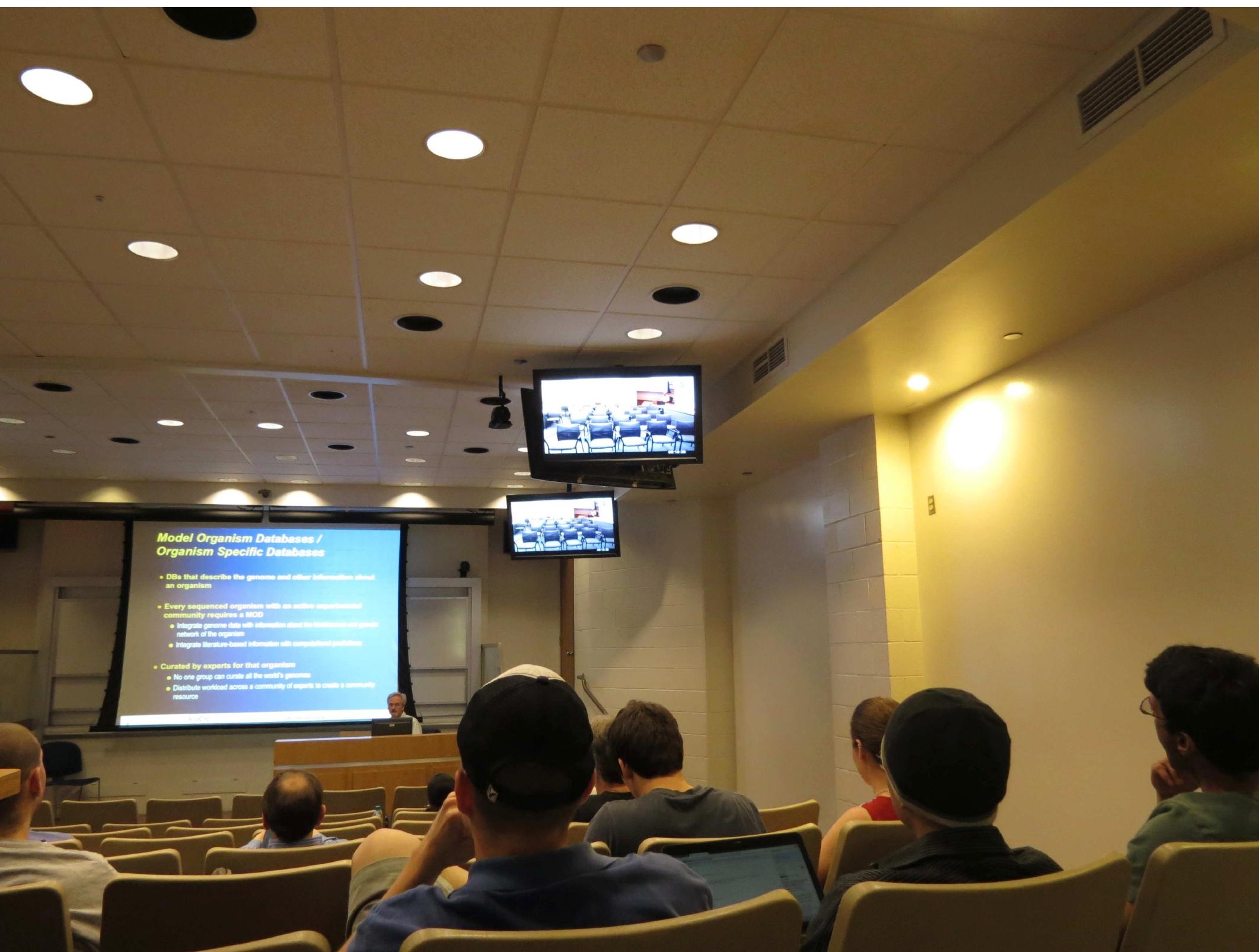




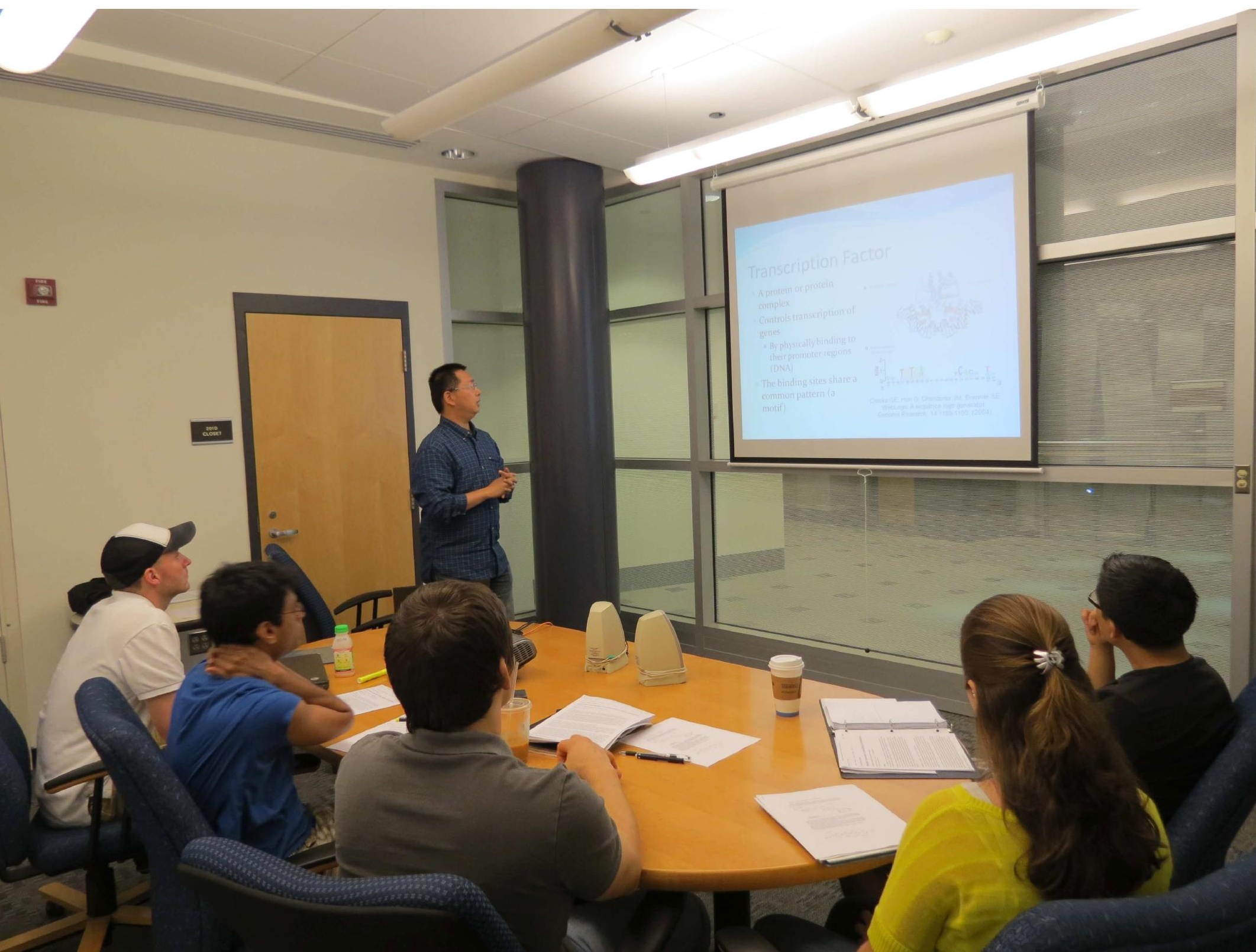




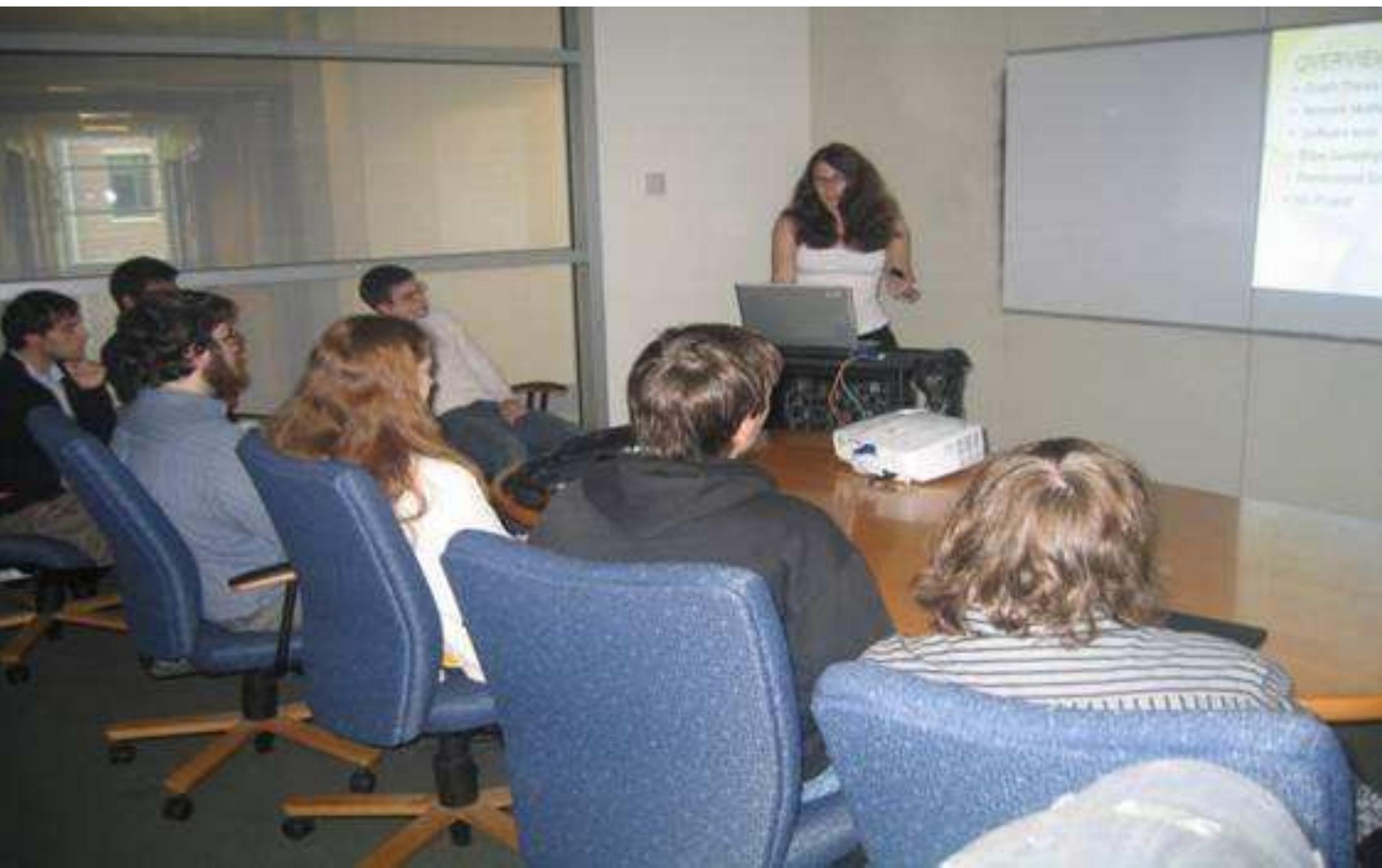




\section{CHUN-HSI HUANG}

\section{COMPUTER SCIENCE \& ENGINEERING}

Phone: (860) 486-5472

E-mail: huang@engr.uconn.edu Website:

http://www.cse.uconn.edu/ huang

\section{Keywords:}

High-Performance Computing, Algorithm Design and Analysis, Computational Biology, Cyber-Infrastructure, Life Science Informatics, Experimental Algorithmics

\section{Summary:}

Chun-Hsi Huang is an Associate Professor with the Department of Computer Science and Engineering at the University of Connecticut. Dr. Huang's research interests are primarily in the areas of High Performance Computing, Computational Biology and Algorithm Design \& Analysis. He is particularly interested in investigating research issues involved in the software/hardware infrastructure, middleware and algorithmic support for extreme-scale computation and information extraction. Recently his research efforts have been on the development, deployment and evaluation of modern large-scale distributed computing infrastructures for this purpose.

Example research foci include the following: (1) exploiting massive parallelism at the algorithm level; (2) coping with software run-time errors from processing elements; (3) efficient large-scale inter-processor and processor-memory communications; and (4) reducing the power requirements for future hardware. 